\title{
Insight into the Pathology of a COL1A1 Signal Peptide Heterozygous Mutation Leading to Severe Osteogenesis Imperfecta
}

\author{
U. Lindert ${ }^{1} \cdot$ M. Gnoli ${ }^{2}$ M. Maioli ${ }^{2} \cdot$ M.F. Bedeschi ${ }^{3} \cdot$ L. Sangiorgi ${ }^{2}$. \\ M. Rohrbach ${ }^{1} \cdot$ C. Giunta ${ }^{1}$
}

Received: 25 September 2017 / Accepted: 30 October 2017/Published online: 3 November 2017

(c) The Author(s) 2017. This article is an open access publication

\begin{abstract}
Osteogenesis imperfecta or "brittle bone disease" is a congenital disorder of connective tissue causing the bone to break easily. Around $85-90 \%$ of cases are due to autosomal dominant mutations in the genes encoding type I collagen, the major organic component of bone. Genotype-phenotype correlations have shown that quantitative defects of collagen type I lead to mild OI, whereas structural defects show a wide clinical range from mild to perinatal lethal. This may partially be explained by the type of amino acid substitution and the relative location in the domain structure. To fully understand the variability of the clinical manifestation and the underlying pathomechanisms, further investigations are required. Here we provide the first biochemical characterization of a mutation at the signal peptide cleavage site of COL1A1, a domain not yet characterized. By steady-state analysis, we observed reduced production of collagen type I. Furthermore, by pulse-chase analysis we detected delayed secretion and partial intracellular retention of collagen I. In the cellular fraction, the electrophoretic migration was abnormal; however, secreted type I collagen showed a normal migration pattern. The intracellular retention of collagen I was confirmed by immunofluorescent staining. Moreover,
\end{abstract}

M. Rohrbach and C. Giunt contributed equally.

\section{Giunta \\ Cecilia.Giunta@kispi.uzh.ch}

1 Connective Tissue Unit, Division of Metabolism and Children's Research Center, University Children's Hospital, Steinwiesstrasse 75, 8032 Zurich, Switzerland

2 Department of Medical Genetics and Skeletal Rare Diseases, Rizzoli Orthopaedic Institute, Bologna, Italy

3 Medical Genetics Unit, Fondazione IRCCS Ca'Granda Ospedale Maggiore Policlinico, Milano, Italy transmission electron microscopy of cultured fibroblasts revealed enlargement of ER cisternae. These results further support the hypothesis that mechanisms interfering with ER integrity play an important role in the pathology of severe OI.

Keywords Osteogenesis imperfecta - Bone · Collagen · COL1A1 - Signal peptide mutation $\cdot$ Signal peptide cleavage site

\section{Introduction}

Osteogenesis imperfecta (OI; MIM 166,200, 1,666,210, 259,420 , and 166,220 ) is a heterogeneous heritable disorder of bone matrix formation and remodeling. The cardinal manifestations are bone fragility and deformity due to hypermineralization of the bone matrix resulting in stiffer bone tissue which is more prone to fractures [1-3]. Based on clinical and radiological findings, individuals with OI are classified according to Sillence as mild type I, perinatal lethal type II, severe type III, or moderately deforming type IV [4]. Around $85-90 \%$ of cases with OI are caused by mutations in the genes COLIAl (MIM 120,150) or COL1A2 (MIM 120,160), encoding type I collagen [5]. Procollagen peptide chains in the rough endoplasmic reticulum (rER) undergo a series of modifications, such as hydroxylation and glycosylation, and assemble into a triple helix consisting of two $\alpha 1$ (I) chains and one $\alpha 2(\mathrm{I})$ chain, respectively. Glycine is required at every third position in the helical part since it is the only amino acid small enough to face into the inner side of the triple helix $[6,7]$. After secretion into the extracellular space and removal of the Nand C-propeptides, the so-formed insoluble mature collagen I molecules can assemble into fibrillar structures. 
Genotype-phenotype correlations have shown that COL1A1 mutations leading to quantitative alterations of the transcript, in particular to haploinsufficiency, are associated with mild OI type I, whereas mutations leading to structural defects result in a wide clinical spectrum from mild to lethal forms. Glycine substitutions in the triple helical part account for the majority of these mutations and are associated with overmodification of the triple helix due to delayed folding. Starting from 2006, several non-collagen genes causing OI have been identified and shown to be involved in collagen I biosynthesis, bone mineralization, and osteoblast development (reviewed in [8]). Unfortunately, the pathophysiological understanding could not keep pace with the expanding insight into the genetic heterogeneity of OI. Therefore, it remains unclear whether common pathological mechanisms exist and whether these are mainly determined by collagen deficiency, cellular malfunction, or structural defects in the extracellular matrix.

Here we provide novel insight into the pathophysiology of OI by investigating a rare disease causing variant localized in the signal peptide of COLIA1 [9], a hitherto functionally uncharacterized domain (Fig. 1a).

A heterozygous de novo p.Gly22Arg mutation at the -1 position of the signal peptide cleavage site was identified in a fetus with severe OI, aborted at the 22nd week of pregnancy (patient ID: AN_000079 in the osteogenesis imperfecta variant database https://oi.gene.le.ac.uk/). The same mutation has been reported previously in a patient with OI type II [10]. Recently the p.Gly22-Gln23del mutation (deletion of the -1 and +1 positions of the signal peptide) has been identified in a patient with OI type III [11]. However, both cases were identified by epidemiological studies and thus no biochemical characterization was performed. Signal peptides contain a hydrophobic core, which allows the targeting of the protein to the ER upon translation, and a cleavage site which is required to release the protein from the membrane pore (translocon). Efficient cleavage by the signal peptidase requires small amino acids (Ala, Gly, Ser) at the -1 position of the cleavage site [12]. To gain insight into the pathomechanism of this form of OI, we set out to analyze collagen biosynthesis and secretion, as well as the cellular ultrastructure.

\section{Methods and Results}

\section{Genetic Analysis}

All coding exons and flanking exon-intron junctions of COL1A1 and COL1A2 were Sanger sequenced using BigDye Terminator version 3.1 cycle sequencing kit and an
ABI Prism 3100 automated DNA sequencer (Applied Biosystems, Foster City, CA).

\section{Cleavage Site Prediction}

In silico analysis of the signal sequence was done by submitting the first 50 amino acids of the COL1A1 sequence to the SignalP 4.1 server at http://www.cbs.dtu. $\mathrm{dk} /$ services/SignalP/ [13].

A strong reduction of the cleavage probability (from 0.956 to 0.319 ) for the p.Gly22Arg substitution was predicted (Fig. 1b).

\section{Cell Culture and Collagen Analyses}

Fibroblasts of the fetus were obtained from an umbilical cord biopsy by explant culture. Patient and control fibroblasts were grown under standard conditions using DMEM medium supplemented with $10 \%$ fetal calf serum, $100 \mathrm{units} / \mathrm{ml}$ of penicillin, $100 \mu \mathrm{g} / \mathrm{ml}$ of streptomycin, and $0.25 \mu \mathrm{g} / \mathrm{ml}$ of amphotericin B (Gibco). Fibroblasts of an unaffected individual in pediatric age were used as a control.

To assess possible effects on collagen biosynthesis and secretion, we performed collagen steady-state and pulsechase analyses in cultured fibroblast as described [14] with minor changes: 4-h pulse labeling of collagen with $\left({ }^{3} \mathrm{H}\right)$ proline and $\left({ }^{3} \mathrm{H}\right)$-glycine was followed by chase with unlabeled amino acids for 5, $30 \mathrm{~min}, 2$ and $24 \mathrm{~h}$.

Steady-state analysis showed that the proportion of type I to type III collagen was reduced in the medium of the OI fibroblasts compared to the control (Fig. 2a).

Pulse-chase analysis showed that the secretion of the $\alpha 1$ (I) and the $\alpha 2$ (I) chains from the cells into the medium was slightly delayed in the OI fibroblasts (Fig. 2b). A small amount of type I collagen was retained in the cells even after $24 \mathrm{~h}$ (arrow, Fig. 2b). Furthermore, in the cell layer, but not in the medium of the OI cells, the $\alpha 1$ (I) and $\alpha 2$ (I) chains appeared as slightly broad and fuzzy bands, suggesting the presence of both overmodified as well as undermodified collagen chains in the 5-min, 30-min, and 2-h chases (Fig. 2b).

\section{Immunofluorescent Staining}

To further investigate the intracellular distribution of collagen in the OI and control fibroblasts, co-immunofluorescent staining for type I collagen (COLI; mouse anticollagen I Abcam ab6308; 1:100) and either the ER marker protein disulfide isomerase (PDI; rabbit anti-PDI Santa Cruz sc-20,132) or the Golgi marker GM130 (rabbit antiGM130 Abcam ab52649; 1:100) was performed as described [14]. Secondary antibodies were goat anti-rabbit 


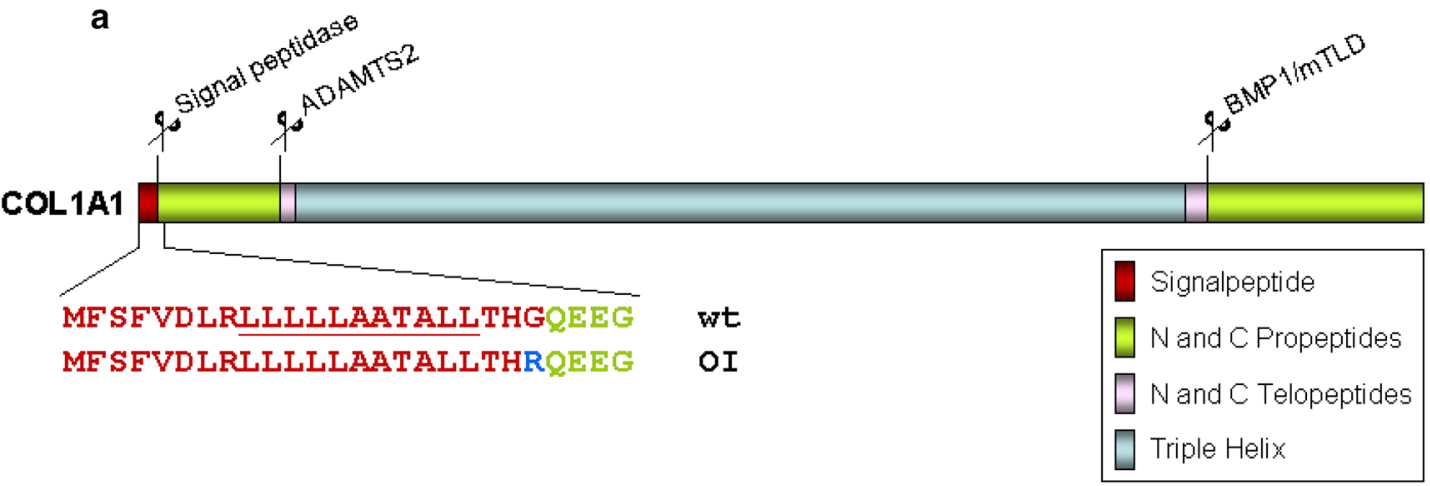

b
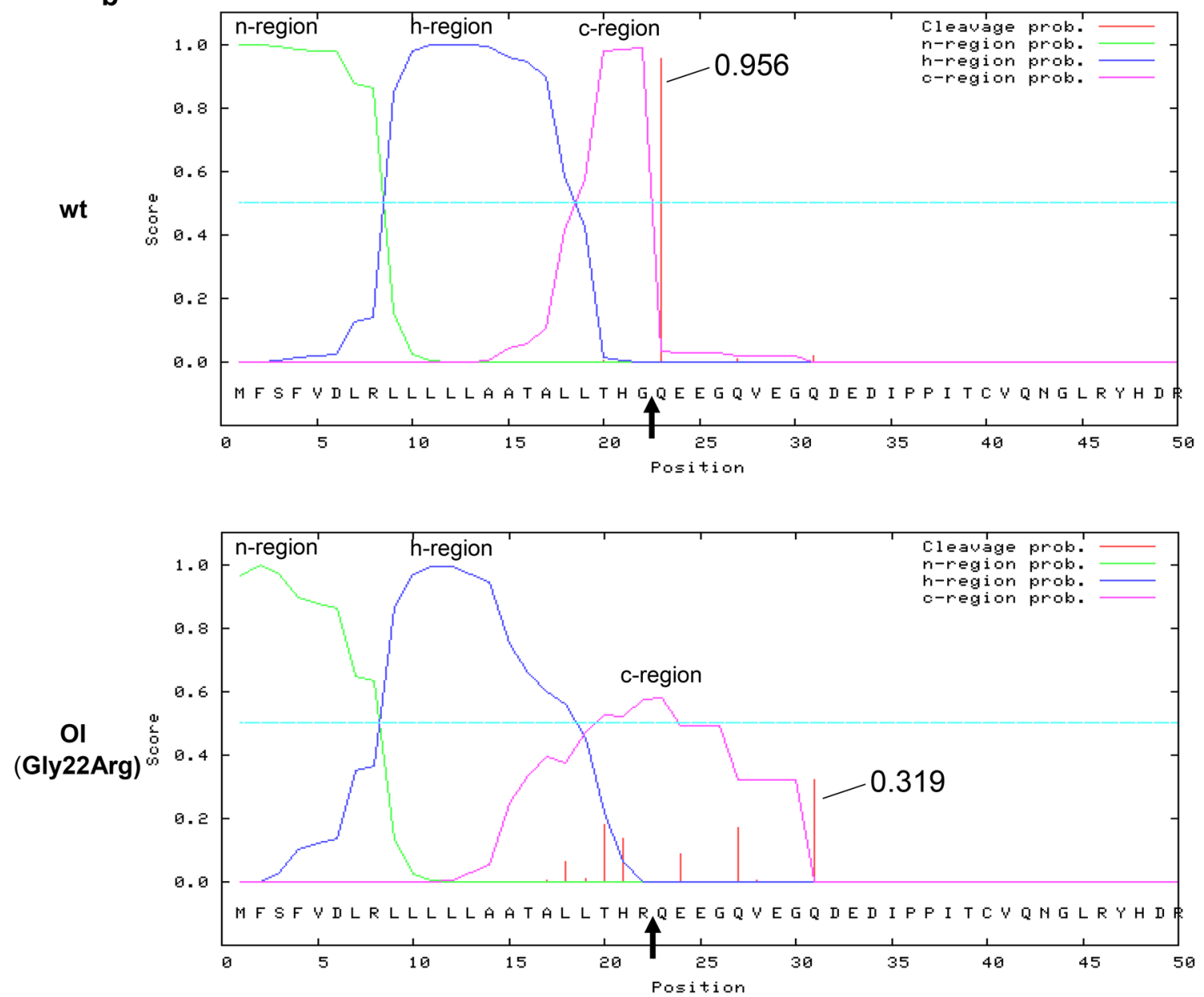

Fig. 1 a Domain structure of COL1A1 protein with p.Gly22Arg substitution in the signal peptide highlighted in blue. $\mathbf{b}$ Prediction of the cleavage site probability of the wt COL1A1 sequence and the p.Gly22Arg substitution using the SignalP algorithm. The algorithm predicts a strong reduction of the cleavage site probability (from 0.956 to 0.319 ) for the p.Gly22Arg substitution. The predicted

AF568 (ab175471) Abcam (1:500) and goat anti-mouse AF488 (A11017) Molecular Probes (1:500). Nuclei were stained with 4',6-diamidino-2-phenylindole (DAPI). Type I collagen localized to the ER as well as to the Golgi in OI as cleavage site for the Gly22Arg substitution with the highest probability is at a different position (between Gly30 and Gln31). A black arrow indicates the position of the wt signal peptide cleavage site; n-region: N-terminal region; h-region: hydrophobic region; c-region: cleavage region (Color figure online)

well as control fibroblasts. Additionally, in the OI fibroblasts, a punctuate staining not overlapping with the ER or Golgi markers was observed (Fig. 2c). 
Fig. 2 Collagen synthesis and secretion in fibroblasts cultures of the OI fetus (OI) and a control (Ctr.); a steady-state analysis showing decreased levels of type I collagen $\alpha 1$ (I) and $\alpha 2(\mathrm{I})$ relative to type III collagen $[\alpha 1(\mathrm{III})]_{3}$ in the medium (M) of the OI fibroblasts suggesting that less collagen type I was produced and secreted by these cells; b pulse-chase analysis showing a decreased amount of type I collagen chains secreted from the cell layer $(\mathrm{C})$ into the medium layer $(\mathrm{M})$ in the OI fibroblasts compared to the control in the 5-min, 30-min, and 2-h chases. Also, bands with a broader migration pattern are visible in the cell layer (C) of the OI fibroblasts (white asterisk) and a small amount of collagen is still visible after 24-h chase in the cell layer of the OI sample (white arrowhead) but not the control; c Co-immunofluorescent staining of type I collagen (COL I) together with ER (PDI) or Golgi (GM130) markers. DAPI was used to stain nuclei and an overlay of COL I with either PDI or GM130 is given showing increased staining for type I collagen in the OI cells and only partial overlap with ER and Golgi markers
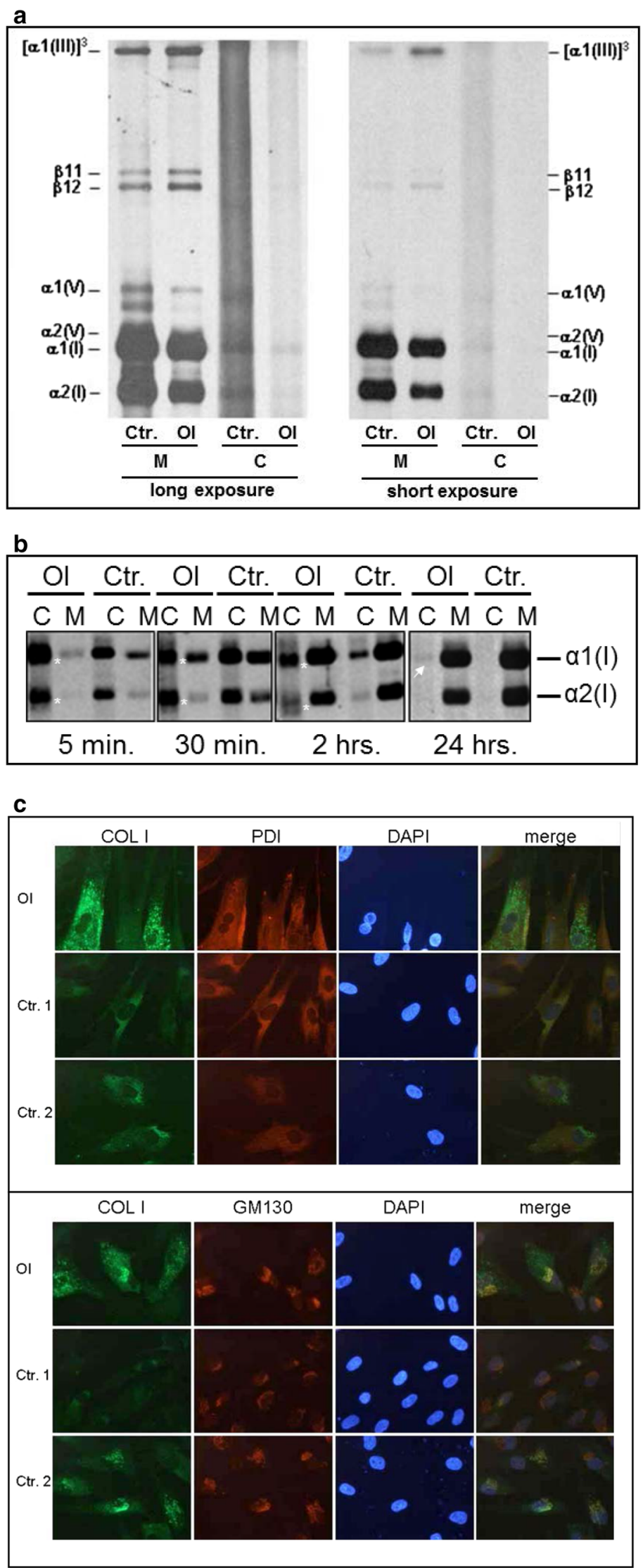


\section{Transmission Electron Microscopy}

To investigate whether the morphology of the ER was altered due to the putative retention of misfolded collagen, we performed transmission electron microscopy on cultured fibroblasts as described [14].

The OI cells (Fig. 3a, b) presented with enlarged cisternae of the rough endoplasmic reticulum (rER) which were not seen in control fibroblasts (Fig. 3c, d).

\section{Discussion}

Here we provide the first biochemical characterization of a mutation in the signal peptide of COL1Al leading to a severe form of $\mathrm{OI}$ and discuss possible underlying pathomechanisms.

Unfortunately, the availability of a single affected subject and the inaccessibility of osteoblasts present a limitation of our study. Despite this drawback, we show that it offers the opportunity to draw general conclusions on the pathomechanism involved in OI.

Firstly, we observed decreased collagen secretion, similar to the findings in OI with haploinsufficiency of COL1A1. However, haploinsufficiency is strongly associated with mild OI suggesting for additional pathological mechanisms responsible for the severe phenotype.
Furthermore, secretion of collagen I by the fibroblasts of the OI fetus was slightly delayed, but both collagen I chains showed a normal migration pattern suggesting that structural alteration of the ECM do not account for the severe phenotype in the fetus. This is in line with the fact that the mutation lies in a domain that is not part of the collagen fibrils in the ECM and also not involved in triple helical folding or processing. Unfortunately, bone tissue for mass spectrometry analysis was not available to definitively rule out the presence of aberrant collagen in the extracellular matrix.

Although the secreted type I collagen showed a normal migration pattern, a portion of collagen with aberrant modification appeared to be retained within the cell. The reduced secretion and aberrant modification of intracellularly retained collagen, not overlapping with the ER-luminal marker, suggest that the p.Gly22Arg mutation impairs cleavage and thus the release of preprocollagen $\alpha 1$ (I) chains from the ER-entry sites (translocon). This is also supported by the results obtained by the in silico prediction of the cleavage efficiency. Impaired release of preprocollagen chains from the translocon might have pleiotropic effects, .e.g., leading to misfolding and ER stress or exhibiting a dominant negative effect on the COL1A1 chain encoded by the normal allele. Furthermore, blocking the translocon might affect cellular functioning since this pore is required for ER entry of other proteins of
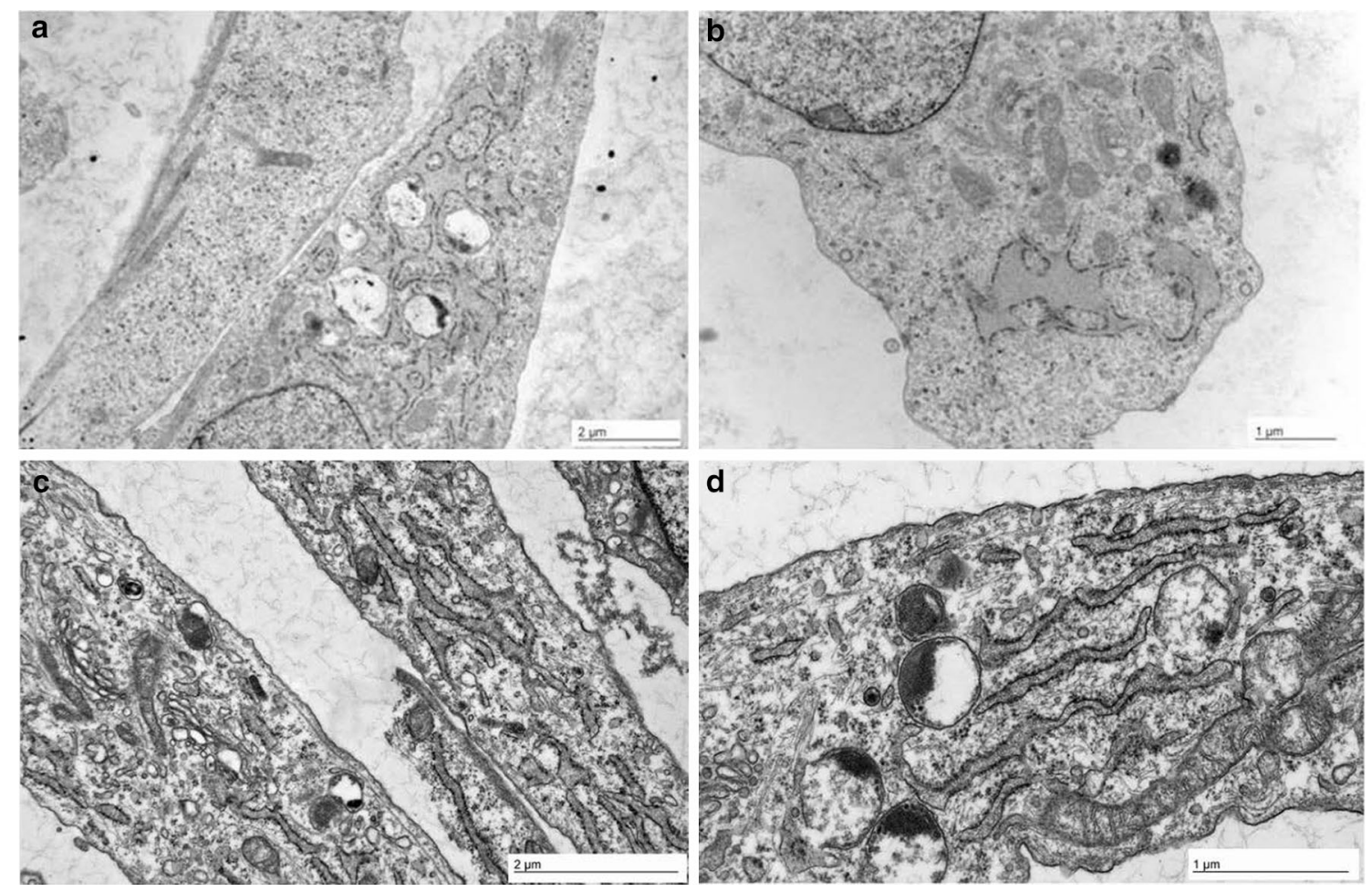

Fig. 3 Transmission electron microscopy (TEM) analysis of cultured fibroblasts showing enlarged ER cisterns in the cells of the fetus (a, b) compared to a control $(\mathbf{c}, \mathbf{d})$ 
the secretory pathway, as well as for the export of misfolded proteins for ERAD or lysosomal degradation. Consistently, we detected dilated ER cisterns by electron microscopy, suggesting that ER stress might be involved in the pathology of OI as shown previously in a study analyzing a G610C mouse model of OI [15].

Pathological effects of signal peptide mutations have been characterized already in other genes (diseases) such as insulin (diabetes), COL10A1 (Schmid-type metaphyseal chondrodysplasia, MCDS), and COL5A1 (classical EhlersDanlos Syndrome, cEDS). These reports have shown that overall, mutations of the hydrophobic region cause protein deficiency as shown for COL5A1 in cEDS, whereas mutations of the cleavage site are associated with ER stress and dominant negative effects on the remaining wild-type protein as demonstrated for COL10A1 in MCDS and insulin in diabetes [16-18]. Thus, our findings are in line with previous reports on pathological effects of cleavage site mutations.

Taken together, our data support the hypothesis that intracellular mechanisms such as accumulation of unfolded proteins and ER stress can contribute to the pathophysiology of the more severe forms of OI (Sillence class II to IV). We suggest, therefore, that therapeutic approaches for OI aiming to increase the clearance of misfolded proteins should be taken seriously into consideration.

Acknowledgements We thank Angelika Schwarze for expert technical assistance with fibroblast cultures. The work has been supported by the Swiss National Science Foundation (SNF-Grant 310030_138288) to CG and MR.

Author Contributions UL wrote the manuscript, and CG and MR participated in drafting the manuscript. UL, CG, and MR contributed to the conception and design of the study. MG, MFB, MM, and LS contributed to the acquisition of biological materials and performed genetic analyses. All authors participated in revising the manuscript and approved the final version of the manuscript for submission.

\section{Compliance with Ethical Standards}

Conflict of interest U. Lindert, M. Gnoli, M. Maioli, MF. Bedeschi, L. Sangiorgi, M. Rohrbach, and C. Giunta declare that they have no conflicts of interest.

Human and Animal Rights and Informed Consent This study was conducted according to the Declaration of Helsinki for Human Rights of 1975 , as revised in 2000, and approved by Swiss Ethics. For the study informed parental consent, in accordance with requirements of the Institutional Ethic Committee of the referring physician (MFB), was obtained.

Open Access This article is distributed under the terms of the Creative Commons Attribution 4.0 International License (http://crea tivecommons.org/licenses/by/4.0/), which permits unrestricted use, distribution, and reproduction in any medium, provided you give appropriate credit to the original author(s) and the source, provide a link to the Creative Commons license, and indicate if changes were made.

\section{References}

1. Marini JC (ed) (2004) Nelson textbook of pediatrics Saunders, Philadelphia

2. Martin E, Shapiro JR (2007) Osteogenesis imperfecta:epidemiology and pathophysiology. Curr Osteoporos Rep 5(3):91-97

3. Phillipi CA, Remmington T, Steiner RD (2008) Bisphosphonate therapy for osteogenesis imperfecta. Cochrane Database Syst Rev 4(4):005088

4. Sillence DO, Senn A, Danks DM (1979) Genetic heterogeneity in osteogenesis imperfecta. J Med Genet 16(2):101-116

5. Marini JC, Forlino A, Cabral WA, Barnes AM, San Antonio JD, Milgrom S, Hyland JC, Korkko J, Prockop DJ, De Paepe A, Coucke P, Symoens S, Glorieux FH, Roughley PJ, Lund AM, Kuurila-Svahn K, Hartikka H, Cohn DH, Krakow D, Mottes M, Schwarze U, Chen D, Yang K, Kuslich C, Troendle J, Dalgleish R, Byers PH (2007) Consortium for osteogenesis imperfecta mutations in the helical domain of type I collagen: regions rich in lethal mutations align with collagen binding sites for integrins and proteoglycans. Hum Mutat 28(3):209-221

6. Bateman JF, Boot-Handford RP, Lamande SR (2009) Genetic diseases of connective tissues: cellular and extracellular effects of ECM mutations. Nat Rev 10(3):173-183

7. Grant Kielty (2002) The Collagen family: structure, assembly, and oragnisation in the extracellular matrix. Connective tissue and its heritable disorders. CV Mosby, St. Louis, pp 159-221

8. Kang H, Aryal ACS, Marini JC (2017) Osteogenesis imperfecta: new genes reveal novel mechanisms in bone dysplasia. Transl Res 181:27-48. https://doi.org/10.1016/j.trsl.2016.11.005

9. Tromp G, Kuivaniemi H, Stacey A, Shikata H, Baldwin CT, Jaenisch R, Prockop DJ (1988) Structure of a full-length cDNA clone for the prepro alpha 1(I) chain of human type I procollagen. Biochem J 253(3):919-922

10. Pollitt R, McMahon R, Nunn J, Bamford R, Afifi A, Bishop N, Dalton A (2006) Mutation analysis of COL1A1 and COL1A2 in patients diagnosed with osteogenesis imperfecta type I-IV. Hum Mutat 27(7):716. https://doi.org/10.1002/humu.9430

11. Lindahl K, Astrom E, Rubin CJ, Grigelioniene G, Malmgren B, Ljunggren O, Kindmark A (2015) Genetic epidemiology, prevalence, and genotype-phenotype correlations in the Swedish population with osteogenesis imperfecta. Eur J Hum Genet 23(8):1112. https://doi.org/10.1038/ejhg.2015.129

12. Choo KH, Ranganathan S (2008) Flanking signal and mature peptide residues influence signal peptide cleavage. BMC Bioinform 9(Suppl 12):S15. https://doi.org/10.1186/1471-2105-9-S12S15

13. Petersen TN, Brunak S, von Heijne G, Nielsen H (2011) SignalP 4.0: discriminating signal peptides from transmembrane regions. Nat Methods 8(10):785-786. https://doi.org/10.1038/nmeth.1701

14. Lindert U, Weis MA, Rai J, Seeliger F, Hausser I, Leeb T, Eyre D, Rohrbach M, Giunta C (2015) Molecular consequences of the SERPINH1/HSP47 mutation in the dachshund natural model of osteogenesis imperfecta. J Biol Chem 290(29):17679-17689. https://doi.org/10.1074/jbc.M115.661025

15. Mirigian LS, Makareeva E, Mertz EL, Omari S, Roberts-Pilgrim AM, Oestreich AK, Phillips CL, Leikin S (2016) Osteoblast malfunction caused by cell stress response to procollagen misfolding in alpha2(I)-G610C mouse model of osteogenesis imperfecta. J Bone Miner Res 31(8):1608-1616. https://doi.org/ 10.1002/jbmr.2824

16. Chan D, Ho MS, Cheah KS (2001) Aberrant signal peptide cleavage of collagen $\mathrm{X}$ in Schmid metaphyseal chondrodysplasia. Implications for the molecular basis of the disease. J Biol Chem 276(11):7992-7997. https://doi.org/10.1074/jbc.M003361200 
17. Liu M, Lara-Lemus R, Shan SO, Wright J, Haataja L, Barbetti F, Guo H, Larkin D, Arvan P (2012) Impaired cleavage of preproinsulin signal peptide linked to autosomal-dominant diabetes. Diabetes 61(4):828-837. https://doi.org/10.2337/db11-0878
18. Symoens S, Malfait F, Renard M, Andre J, Hausser I, Loeys B, Coucke P, De Paepe A (2009) COL5A1 signal peptide mutations interfere with protein secretion and cause classic Ehlers-Danlos syndrome. Hum Mutat 30(2):E395-E403. https://doi.org/10. 1002/humu.20887 